\title{
Dark Field STEM Imaging of Isolated Heavy Atoms in a Transition Metal Matrix
}

\author{
M.D. Shannon* and G.B. Shannon** \\ * UK SuperSTEM Facility, Keckwick Lane, Daresbury, WA4 4AD, UK and Department of \\ Engineering, University of Liverpool, Brownlow Hill, Liverpool \\ ** Department of Pharmacy, University of Nottingham UK
}

Co is a common catalyst for the Fischer-Tropsch process which synthesises liquid paraffins from $\mathrm{CO}$ and $\mathrm{H} 2$. The metal particles are typically a few $\mathrm{nm}$ in size supported on an oxide such as $\gamma$-alumina. The alumina is impregnated with a salt of $\mathrm{Co}$ and calcined in air to form the spinel Co3O4. The Co particles are then produced by reduction of this oxide in $\mathrm{H} 2$ in two steps [1]. To maximize the surface area for reaction, promoters such as $\mathrm{Re}, \mathrm{Pt}$, Ir or $\mathrm{Ru}$ are added. These lower the temperatures at which each reduction step occurs and thereby inhibit sintering. However a typical recipe might involve $20 \mathrm{wt} \%$ Co and $1 \mathrm{wt} \%$ of the PGM (platinum group metal). This is very costly. As part of a study to understand how the promotion works with a view to lowering the PGM content, HAADF imaging on the aberration-corrected VG501 STEM (Nion-corrector) at SuperSTEM was used to attempt to image the positions of the promoter atoms after full reduction [1]. Not all the expected PGM atoms were observed and there are several possible reasons for this. However, of these the one that fundamentally undermines the experiment is the physics of the electron interactions with the PGM atoms in a relatively heavy matrix.

Multislice simulations including thermal diffuse scattering using the codes of Kirkland [2] and Koch [3] showed that for the standard conditions on this instrument $(100 \mathrm{keV}$, convergence angle $24 \mathrm{mrad}$, HAADF detector inner angle $70 \mathrm{mrad}$ and outer angle $210 \mathrm{mrad}$ ) the contrast of the PGM atoms was a very strong function of its depth within the crystal relative to the incident beam [1]. Two models of FCC Co were generated; one a parallel sided foil and the other a cube-octahedral particle about $3.5 \mathrm{~nm}$ in size. Re and $\mathrm{Ru}$ were chosen to represent the promoter atoms. For the foil a single dopant (promoter) atom was positioned at a number of depths whilst parameters such as Cs and defocus were varied.

The visibility of the dopant atoms is not at the optimum focus for the Co crystal itself as shown in Fig 1 where a dopant atom is in the exit surface of a 29 layer crystal in [110] orientation. For the conditions given the Co lattice is in sharpest focus at $5.5 \mathrm{~nm}$ defocus whilst the Re is most readily detected when the Co lattice contrast is minimal at $-4-6 \mathrm{~nm}$.

The effect of the depth of the dopant is shown in Fig 2(a). Each column contains 8 atoms. Columns 1, 2, 3 and 8 contain Co only whilst, in the direction of the incident beam, Co atoms 6, 7, 3 and 3 of columns 4, 5, 6 and 7 respectively are replaced by Re. Clearly those nearest the exit surface exhibit highest contrast. Indeed $2 \mathrm{Re}$ atoms in a column in positions 1 and 2 would show less contrast than 1 atom in position 7 and a single $\mathrm{Re}$ atom in position 1 would be barely detectable. The contrast variation with depth is useful provided the number of heavy atoms in a column is known. Recent simulations involving more careful consideration of the inner and outer angles of the annular dark field detector suggest that it is possible to design an experiment in which the number of dopant atoms in a column dominates the intensity an there is a much smaller depth variation (Fig 2(b). This 
signal and the standard HAADF signal may then be combined to estimate the positions of dopant atoms at least in those containing just one. How to achieve this will be discussed in the presentation. It should be noted that these effects are related to the relatively high atomic number of the Co matrix.

\section{References}

[1] M.D. Shannon, C.M. Lok and J.L. Casci, J. Catal. 249 (2007) 41.

[2] E.J. Kirkland, Advanced Computing in Electron Microscopy, Plenum, New York 1998.

[3] C. Koch, Ph.D. Thesis, Arizona State University, 2002.

[4] Funding was provided by the Royal Society through an Industrial Fellowship (MDS) and by the University of Liverpool (GBS). MDS was on secondment from ICI plc and later Intertek plc.

\section{Dopant in Exit Surface: Effect of Focus}

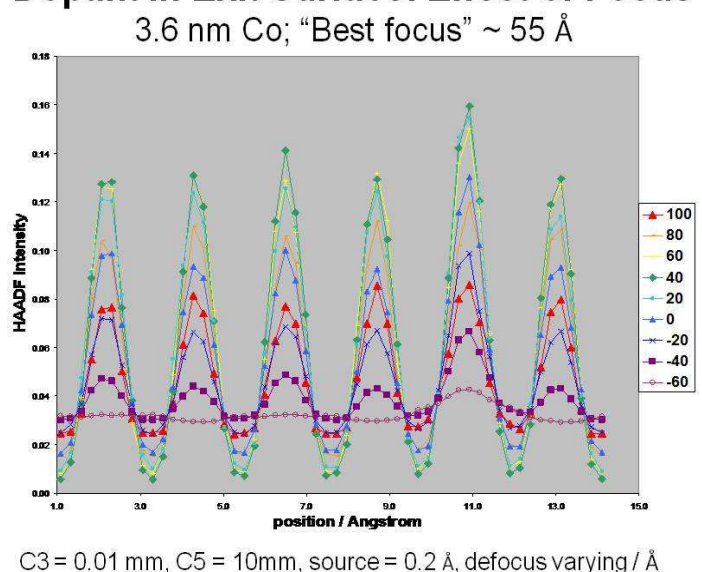

Fig 1. HAADF line profile from Co [110] projection; 15 Co atoms in each column except for $5^{\text {th }}$ column which has $14 \mathrm{Co}$ and $1 \mathrm{Re}$ in the exit surface.

Fig 2. (a) HAADF line profile with standard annular detector and (b) special detector.
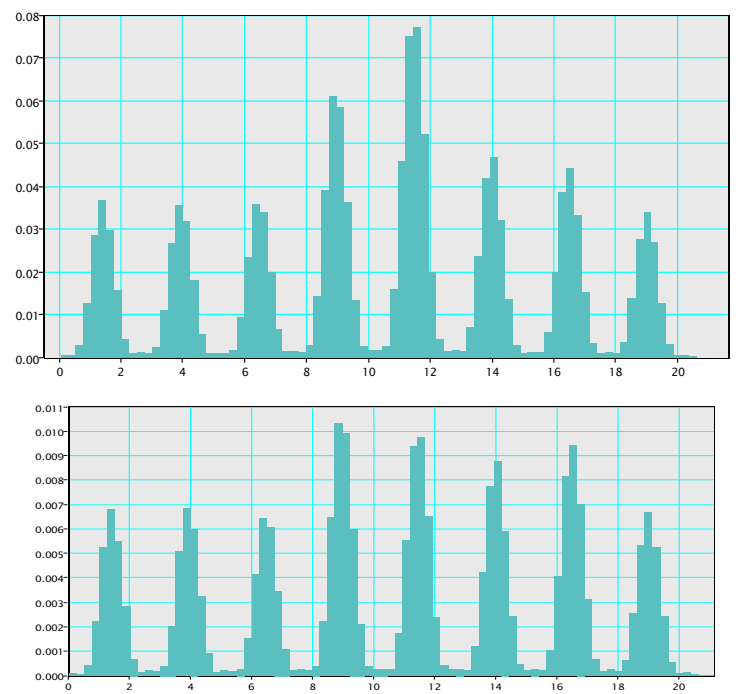